\title{
Temperature dependence of the picosecond spin Seebeck effect ${ }^{\circ}$
}

Cite as: Appl. Phys. Lett. 119, 032401 (2021); https://doi.org/10.1063/5.0050205

Submitted: 12 March 2021 • Accepted: 02 July 2021 • Published Online: 19 July 2021

Farhan Nur Kholid, Dominik Hamara, Marc Terschanski, et al.

\section{COLLECTIONS}

EP

This paper was selected as an Editor's Pick
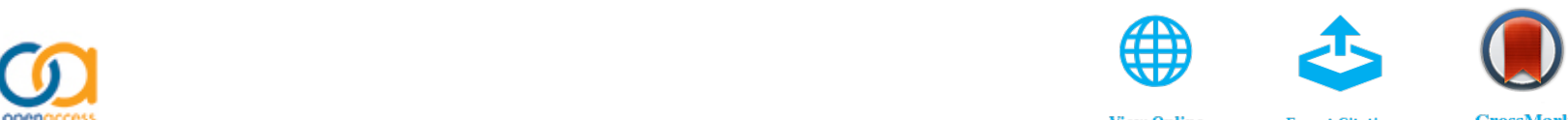

\section{ARTICLES YOU MAY BE INTERESTED IN}

Time-resolved measurement of magnetization vectors driven by pulsed spin-orbit torque Applied Physics Letters 119, 022404 (2021); https://doi.org/10.1063/5.0055458

Strong magnon-photon coupling with chip-integrated YIG in the zero-temperature limit Applied Physics Letters 119, 033502 (2021); https://doi.org/10.1063/5.0054837

Current-induced magnetization switching at charge-transferred interface between topological insulator ( $\mathrm{Bi}, \mathrm{Sb})_{2} \mathrm{Te}_{3}$ and van der Waals ferromagnet $\mathrm{Fe}_{3} \mathrm{GeTe}_{2}$ Applied Physics Letters 119, 032402 (2021); https://doi.org/10.1063/5.0057863

耳QBLOX

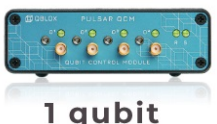

Shorten Setup Time Auto-Calibration More Qubits

Fully-integrated Quantum Control Stacks Ultrastable DC to $18.5 \mathrm{GHz}$ Synchronized $<<1$ ns Ultralow noise

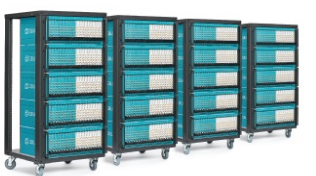

100s qubits

visit our website > 


\title{
Temperature dependence of the picosecond spin Seebeck effect (ip
}

\author{
Cite as: Appl. Phys. Lett. 119, 032401 (2021); doi: 10.1063/5.0050205 \\ Submitted: 12 March 2021 - Accepted: 2 July 2021 . \\ Published Online: 19 July 2021
}

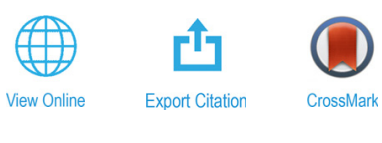

\begin{abstract}
Farhan Nur Kholid, ${ }^{1,2}$ (D) Dominik Hamara, ${ }^{1}$ iD Marc Terschanski, ${ }^{3}$ Fabian Mertens, ${ }^{3}$ iD Davide Bossini, ${ }^{4}$ Mirko Cinchetti, ${ }^{3}$ (D) Lauren McKenzie-Sell, ${ }^{7,5}$ (iD James Patchett, ${ }^{1}$ (D) Dorothée Petit, Russell Cowburn, Jason Robinson, ${ }^{5}$ (D) Joseph Barker, ${ }^{6}$ (D) and Chiara Ciccarelli ${ }^{1, a)}$ (iD
\end{abstract}

\author{
AFFILIATIONS \\ ${ }^{7}$ Cavendish Laboratory, University of Cambridge, Cambridge CB3 OHE, United Kingdom \\ ${ }^{2}$ Physics Department, Lancaster University, Lancaster LA1 4YB, United Kingdom \\ ${ }^{3}$ Experimentelle Physik VI, TU Dortmund, Otto-Hahn-Strasse 4, D-44227 Dortmund, Germany \\ ${ }^{4}$ Department of Physics and Center for Applied Photonics, University of Konstanz, Universitätstraße 10, 78457 Konstanz, Germany \\ ${ }^{5}$ Department of Materials Science and Metallurgy, 27 Charles Babbage Rd., Cambridge CB3 OFS, United Kingdom \\ ${ }^{6}$ School of Physics and Astronomy, University of Leeds, Leeds LS2 9JT, United Kingdom \\ ${ }^{a}$ Author to whom correspondence should be addressed: cc538@cam.ac.uk
}

\begin{abstract}
We performed temperature-dependent optical pump-THz emission measurements in $\mathrm{Y}_{3} \mathrm{Fe}_{5} \mathrm{O}_{12}$ (YIG)|Pt from $5 \mathrm{~K}$ to room temperature in the presence of an externally applied magnetic field. We study the temperature dependence of the spin Seebeck effect and observe a continuous increase as temperature is decreased, opposite to what is observed in electrical measurements, where the spin Seebeck effect is suppressed as $0 \mathrm{~K}$ is approached. By quantitatively analyzing the different contributions, we isolate the temperature dependence of the spin-mixing conductance and observe features that are correlated with the bands of magnon spectrum in YIG.
\end{abstract}

(C) 2021 Author(s). All article content, except where otherwise noted, is licensed under a Creative Commons Attribution (CC BY) license (http:// creativecommons.org/licenses/by/4.0/). https://doi.org/10.1063/5.0050205

The longitudinal spin Seebeck effect (LSSE) ${ }^{1}$ describes the transfer of a spin current from a magnetic insulator (MI) driven by a temperature gradient. An adjacent heavy metal (HM) layer with large spin-orbit coupling is typically used to convert the spin current into an electrical signal via the inverse spin Hall effect (ISHE). ${ }^{2,3}$ The LSSE has been measured in a variety of different materials such as ferromagnets, ${ }^{1,4,5}$ anti-ferromagnets, ${ }^{6,7}$ and paramagnets. ${ }^{8}$ Magnetic insulators (MI) such as $\mathrm{Y}_{3} \mathrm{Fe}_{5} \mathrm{O}_{12}$ (yttrium iron garnet-YIG) are particularly interesting for studies on the LSSE since the absence of electron charge transport allows the roles of magnons and phonons to be identified in the spin transfer. ${ }^{1,3,9,10}$ Temperature, thickness, and magnetic field dependence studies have contributed to a phenomenological picture of the magnon-driven spin current. ${ }^{11-15}$ A temperature gradient across the magnetic insulator thickness leads to the diffusion of thermal magnons that accumulate at the interface with the HM. ${ }^{16,17}$ The temperature dependence of the magnon propagation length $\lambda_{\mathrm{m}}$ results in a characteristic peak in the SSE signal at low temperature when the thickness of the MI is comparable to $\lambda_{\mathrm{m}} \cdot{ }^{12}$ Low frequency magnons play a dominant role due to their large population and longer thermalization lengths. Their contribution can be suppressed by large magnetic fields, which raise the energies of the magnon spectrum. ${ }^{14,15}$

This picture of a bulk-like transport induced by a temperature gradient picks up the essential features of the LSSE. However, several experimental results raise questions on the details of how the spin current is transferred across the $\mathrm{MI} \mid \mathrm{HM}$ interface. ${ }^{12}$ This contribution has been challenging to isolate in electrical measurements of the LSSE, and its temperature dependence is not known.

Recently, ultra-fast experimental techniques using femtosecond lasers have enabled the study of the LSSE and the underlying physical mechanisms of spin current generation at picosecond and shorter timescales. ${ }^{18,19}$ In these experiments, a laser pulse rapidly heats the free electrons in the HM, quickly thermalizing to an effective temperature, $T_{\mathrm{e}}$. The temperature of the magnons in the insulator, $T_{\mathrm{m}}$, is increased primarily by the spin current, which propagates across the interface from the hotter metal. This thermalization process is proportional to $T_{\mathrm{e}}-T_{\mathrm{m}}$, and its timescale is ultimately determined by the electron-magnon scattering time. ${ }^{18}$ In this ultra-short time window after the laser excitation, a thermal gradient is not yet established in 
the bulk of the MI, and the spin current generation originates only at the interface between the MI and the HM. ${ }^{19}$

In this study, we measured the LSSE in YIG|Pt on the picosecond timescale in the low temperature range from $5 \mathrm{~K}$ to room temperature. We observed a different temperature dependence of the LSSE compared to DC electrical studies carried out in the same temperature range. ${ }^{12,14,15}$ Our sample is a $100 \mathrm{~nm}$ thick commercial YIG film grown by liquid phase epitaxy on a (111)-oriented $\mathrm{Gd}_{3} \mathrm{Ga}_{5} \mathrm{O}_{12}$ (GGG) substrate. We cleaned the surface using piranha etching and then sputtered a $5 \mathrm{~nm}$ thick layer of Pt on top. Figure 1 shows the two different orientations of our experiments. We pump the sample from either the GGG side or the Pt side with 50 fs laser pulses with a central wavelength of $800 \mathrm{~nm}$. Any spin transfer across the YIG|Pt interface triggered by the pump pulse is converted into an electric current via the inverse spin-Hall effect in the Pt layer. This produces a broad-band electric-dipole emission $E_{\mathrm{THz}}(\omega)$ with a bandwidth directly related to the Fourier transform of the spin current $j_{\mathrm{s}}(\omega)$ as $^{20}$

$$
\mathrm{E}_{\mathrm{THz}}(\omega)=\frac{Z_{0}}{n_{\mathrm{YIG}}(\omega)+n_{0}(\omega)+\int_{0}^{d} Z_{0} \sigma_{\mathrm{Pt}}(\omega) \mathrm{d} z} \frac{\lambda_{\mathrm{s}} \Theta_{\mathrm{SH}} \mathrm{e}_{\mathrm{s}}(\omega)}{\hbar},
$$

where $Z_{0}$ is the free space impedance in $\Omega, \hbar$ is Planck's constant, $e$ is the charge of an electron, $\lambda_{\mathrm{s}}, \sigma_{\mathrm{Pt}}, d$, and $\Theta_{\mathrm{SH}}$ are, respectively, the spin diffusion length in $\mathrm{nm}$, the electrical conductivity in $\Omega^{-1} \mathrm{~cm}^{-1}$, the thickness in $\mathrm{nm}$, and the spin-Hall angle of the Pt layer. $n_{\mathrm{YIG}}(\omega)$ and $n_{0}(\omega)$ represent the refractive indices of YIG and air. The emitted radiation $S(t)$ is detected in the time-domain by electro-optic sampling with a 1-mm thick ZnTe crystal, and its Fourier transform is given by the convolution of $\mathrm{E}_{\mathrm{THz}}(\omega)$ [Eq. (1)] with the detector response function, which is bandwidth limited to the $0.2-2.5 \mathrm{THz}$ range. We apply an external magnetic field $\left(\mu_{0} H= \pm 0.5 \mathrm{~T}\right)$ along the [100] direction (Fig. 1) during the measurements to saturate the YIG magnetization. We extract an odd-in-magnetic field $S_{-}(t)=[S(t,+H)-S(t,-H)] / 2$ and an even-in-magnetic field $S_{+}(t)=[S(t,+H)+S(t,-H)] / 2$ contribution to the overall emission. $S_{+}$and $S_{-}$label the peak value of $S_{+}(t)$ and $S_{-}(t)$, respectively. $S_{+}(t)$ is polarized in the [100]-[010] plane [Figs. 2(a) and 2(b)]. Its dependence on the pump polarization
[Fig. 2(b)] connects its origin to optical rectification. Both bulk GGG and YIG are centrosymmetric. ${ }^{21,22}$ However, their lattice mismatch induces elastic deformations in YIG close to the interface that gradually changes its lattice parameters, breaking inversion symmetry and yielding a non zero value for the second order electro-optic constant $\chi^{(2)}$, as also confirmed by the measurement of optical second harmonic generation. ${ }^{23}$ From this point forward, we focus on the $S_{-}(t)$ contribution that is due to the LSSE. Unlike $S_{+}(t), S_{-}(t)$ does not show any dependence on pump polarization and is always polarized along the [010] axis, perpendicular with respect to the interface normal and the YIG magnetization [Fig. 2(b)]. The reversal of the interface normal vector with respect to the pump pulse propagation direction results in a polarity switching of the emitted THz radiation [Fig. 2(c)]. Both observations are consistent with the symmetry of the ISHE for a spin current traveling across the interface with spin polarization along the [100] direction. ${ }^{2}$ As a function of the external magnetic field, $S_{-}$follows the hysteresis curve of the YIG magnetization [Fig. 2(d)], also in agreement with previous electrical and optical measurements of the LSSE., ${ }^{5,18}$

Figure 3(a) shows the temperature dependence of $S_{-}$. The continuous line represents a fitting with the function $\left(T_{\mathrm{C}}-T\right)^{\alpha}$, where $\mathrm{T}_{\mathrm{C}}=550 \mathrm{~K}$ is the Curie temperature and $\alpha=2.9 \pm 0.1$. This trend is similar to the temperature dependence measured above room temperature with both low-frequency electrical ${ }^{11}$ and ultra-fast optical methods $^{18}$ but is remarkably different from the low temperature behavior of the LSSE measured in adiabatic conditions, where the signal diminishes toward $0 \mathrm{~K}^{12,14}$ The fact that our LSSE signal is not suppressed at $0 \mathrm{~K}$ excludes that thermal magnons are the main carriers of the spin current. In our experiment, we detect the spin current generated in a time interval up to a few picoseconds after laser absorption. This interval is orders of magnitude shorter than the time needed to establish a thermal gradient in bulk YIG (1-100 ns). ${ }^{24,25}$ When the laser pulse hits the sample, most of the energy is absorbed by the Pt layer. While Pt has a strong optical absorption $\left(\sim 10^{7} \mathrm{~cm}^{-1}\right),{ }^{28}$ enhanced by the Etalon effect, ${ }^{28}$ the absorption in GGG|YIG $\left(10 \mathrm{~cm}^{-1}\right)$ is essentially negligible. ${ }^{29,30}$ The electrons in Pt are heated within a few tens of femtoseconds. ${ }^{18}$ At the short timescales after laser absorption $(\sim 1 \mathrm{ps})$ probed in our measurement, thermalization of these hot electrons

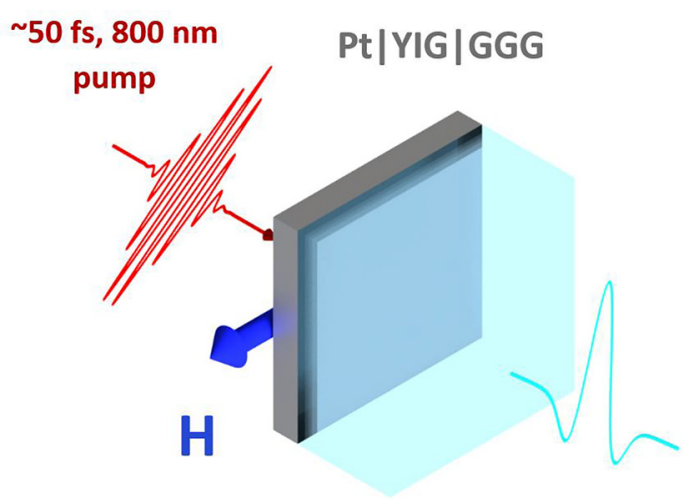

Broadband $\mathrm{THz}$ emission

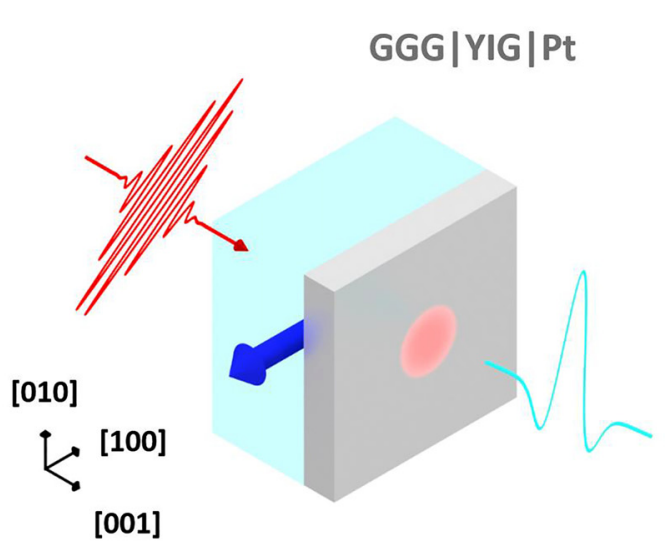

FIG. 1. Schematic illustration of the experiment performed with the femtosecond laser pulses incident on the Pt side (left) and the GGG substrate side (right). 

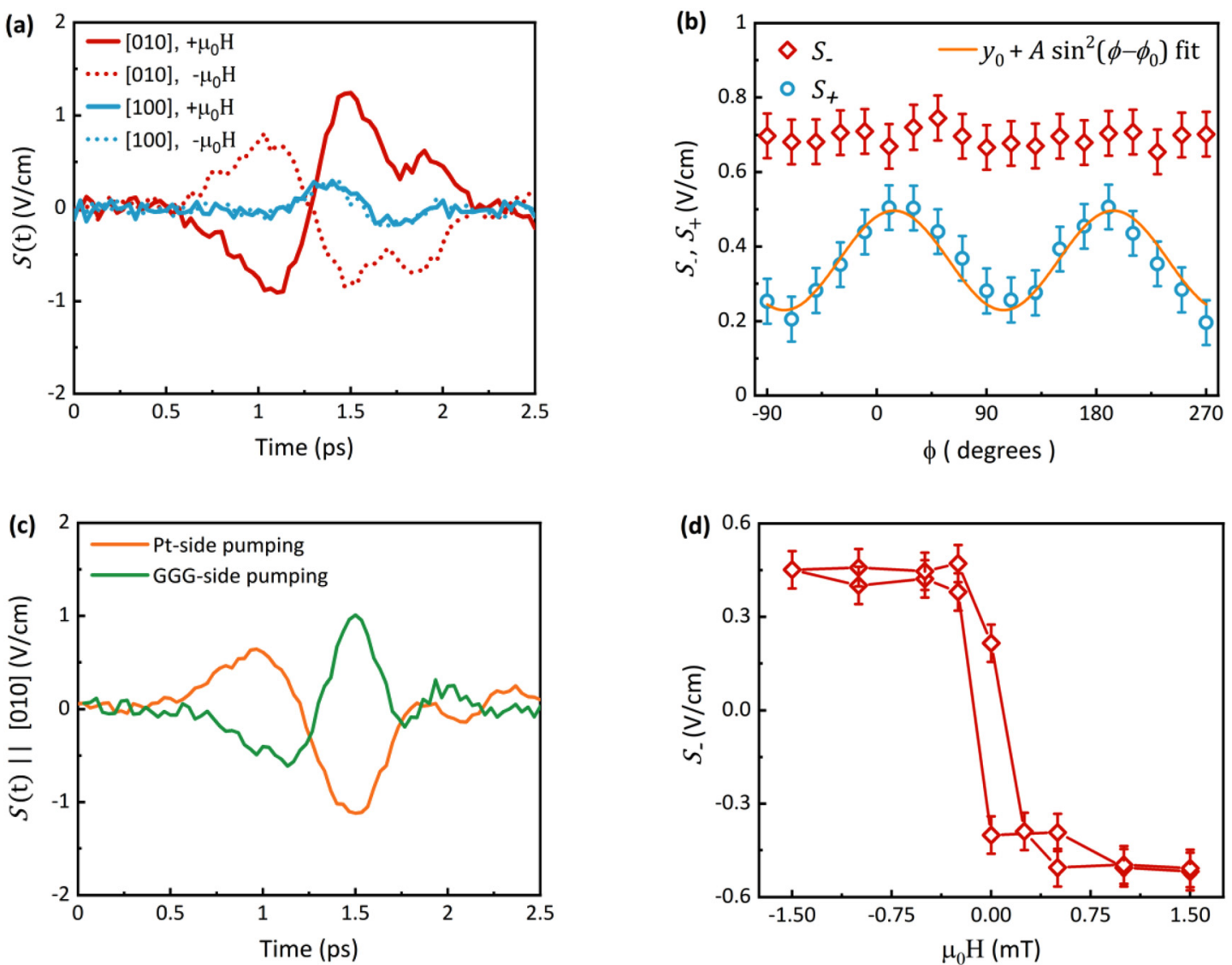

FIG. 2. (a) Time-domain THz emission resolved along the [100]- and [010]-axis, measured at $10 \mathrm{~K}$. Odd-in-field signal $S_{-}$only appears along the [010]-axis component, whereas the signal along the [100]-axis is even-in-field $S_{+}\left(\mu_{0} \mathrm{H}= \pm 0.5 \mathrm{~T}\right)$. (b) $S_{+}$(blue circle) and $S_{-}$(red diamond) dependence on the linear pump polarization where the angle $\phi$ is relative to the [010]-axis. These measurements were carried out for signals along the [010]-axis at room temperature. The orange line is a fit using $y_{0}+A \sin ^{2}\left(\phi-\phi_{0}\right)$, where $y_{0}$ is a constant offset, $A$ is the magnitude of the optical rectification signal, and $\phi_{0}$ is an angle offset. This angular dependence agrees with the second harmonic generation measurement in $\mathrm{GGG} / \mathrm{YIG}^{23}$. An offset of $-0.3 \mathrm{~V} / \mathrm{cm}$ is applied to $S_{+}$for clarity. (c) $E_{\mathrm{THz}}$ polarized along the [010]-axis in the time-domain for Ptside and GGG-side pumping. (d) Hysteresis curve of $S_{-}$measured at room temperature.

mainly occurs via two mechanisms. The first mechanism is electronphonon scattering within $\mathrm{Pt}$, which does not lead to any energy transfer across the interface but determines the time evolution of the electron temperature in Pt. The second mechanism is electronmagnon scattering via inelastic spin-flip processes, which is the origin of the spin transfer across the interface.

Energy transfer across the interface will also occur via the phonon-phonon interaction, but this is a slower process that we can ignore $^{19}$ and we can assume that the temperature of the YIG lattice coincides with the ambient temperature at picosecond timescales.

In our measurement of the LSSE, we are, thus, probing the electron-magnon interactions localized at the interface. The interfacial spin transport parameters are summarized by the spin-mixing conductance $g^{\uparrow \downarrow}$, and the resulting spin current can be written as ${ }^{17,26}$

$$
j_{\mathrm{s}}=\frac{\gamma \hbar k_{\mathrm{B}} g^{\uparrow \downarrow}}{2 \pi M_{\mathrm{s}} V}\left(T_{\mathrm{e}}-T_{\mathrm{m}}\right),
$$

where $\gamma$ is the gyromagnetic ratio, $k_{\mathrm{B}}$ is the Boltzmann constant, $M_{\mathrm{s}}$ is the saturation magnetization of YIG, and $V$ is the unit cell volume. In the case of a femtosecond laser excitation, $\Delta \mathrm{T}_{\mathrm{em}}=\mathrm{T}_{\mathrm{e}}-\mathrm{T}_{\mathrm{m}}$ is set by the energy deposited in the HM layer, in other words by the absorbed laser fluence. This equation is strictly derived in the DC limit; however, in generalizations that allow for a non-equilibrium electron and magnon distribution, the relevant physics is contained in an interface electron-magnon scattering contribution, which effectively modifies the zero temperature value of the spin mixing conductance. ${ }^{31}$ It is this electron-magnon interfacial scattering we are probing in this experiment, which we describe as the temperature dependence of the spin mixing conductance.

To understand the origin of the temperature dependence of the picosecond LSSE in Fig. 3(a), we consider all parameters that contribute to its magnitude, as expressed in Eqs. (1) and (2). In Fig. 3(b), we plot $S_{-}$normalized by the inverse of YIG magnetization $1 / \mathrm{M}_{\mathrm{s}}$ as measured by SQUID [Fig. 3(a)], which shows that $M_{\mathrm{s}}$ is not accountable for the large change in the $\mathrm{THz}$ emission [see Eq. (2)]. To experimentally verify how $\Delta T_{\mathrm{e}}$ is influenced by the ambient temperature, we perform the pump-probe transient reflectivity measurement on glass $\mid \mathrm{Pt}$ bilayers from 10 to $300 \mathrm{~K}$. The transient change in reflectivity 

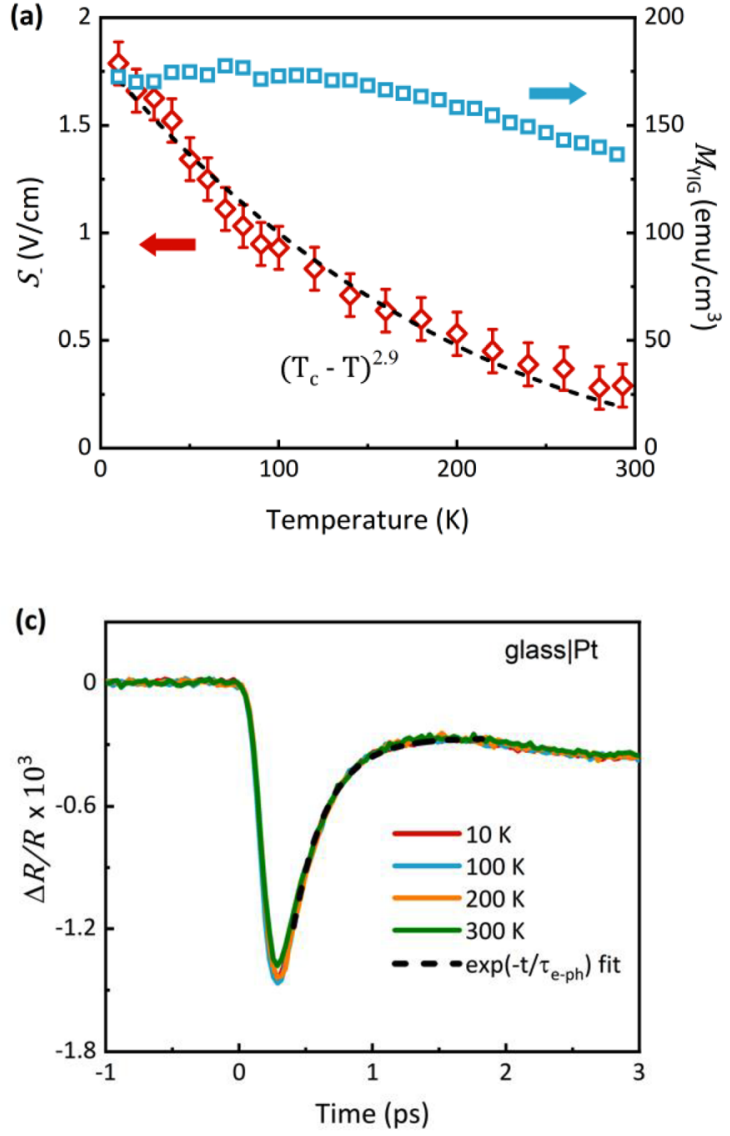
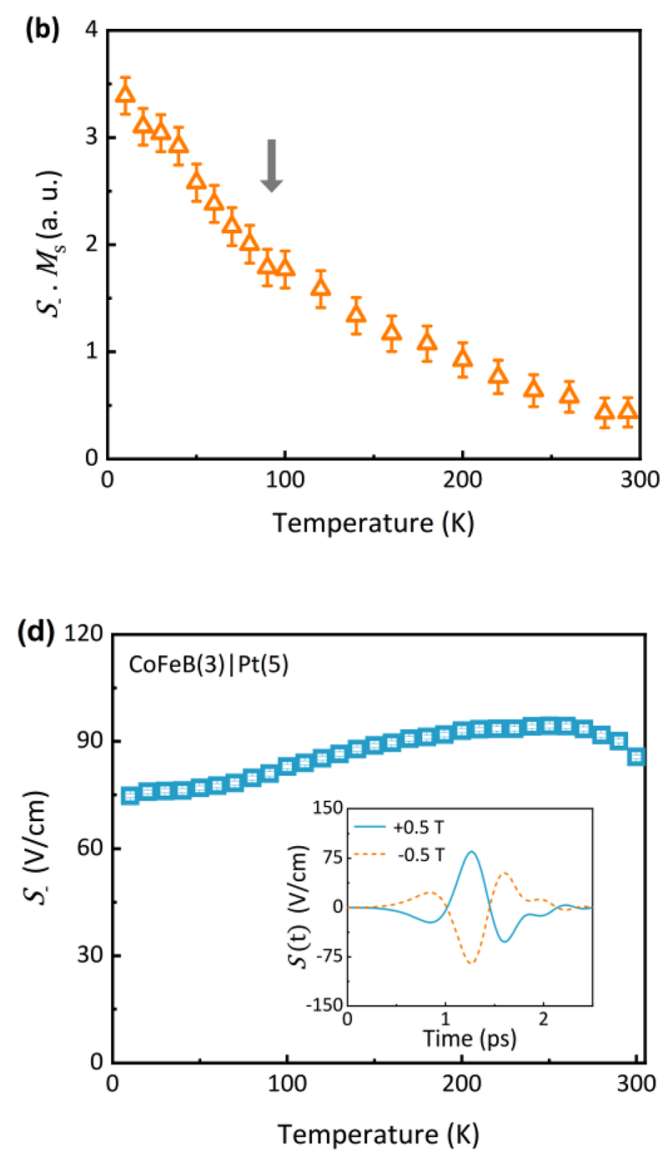

FIG. 3. (a) Temperature dependence of $S_{-}$. The dashed line is a fit with a function $A\left(T_{C}-T\right)^{\alpha}, A=1.5 \pm 1.3, \alpha=2.9 \pm 0.1$, and $T_{C}=550 \mathrm{~K}$. (b) Temperature dependence of the normalized $S_{-}$with the inverse of YIG magnetization $1 / M_{s}, S_{-} . M_{s}$. The gray arrow indicates the temperature at which the slope $d S_{-} / d T$ changes. (c) Time-resolved transient reflectivity $(\Delta R / R)$ of glass $\mid \mathrm{Pt}$ measured in a temperature range of $10-300 \mathrm{~K}$ at a fixed pump fluence of $0.4 \mathrm{~mJ} / \mathrm{cm}^{2}$. The dashed line is an exponential fit $\propto \exp \left(-\frac{t}{\tau_{e-p h}}\right)$. (d) Peak THz emission from CoFeB $(3 \mathrm{~nm}) \mid \mathrm{Pt}(5 \mathrm{~nm})$ as a function of ambient temperature, where the pump pulse hits from the CoFeB side. The error bar is comparable with the symbol size. The inset shows the time-domain data for opposite field polarities $\pm 0.5 \mathrm{~T}$.

$\Delta R / R(t)$ is proportional to the electron temperature increase $\Delta T_{\mathrm{e}}(t) .{ }^{32,33}$ As seen in Fig. 3(c), the peak magnitude of $\Delta R / R$ is weakly affected by decreasing the ambient temperature within the time resolution of the transient reflectivity measurements $(\sim 100 \mathrm{fs}$, determined by the pulse width), which was previously observed in other transition metals. ${ }^{34}$ In the supplementary material, we show that $\Delta R / R$ only weakly depends on ambient temperature at the low pump fluences used for the $\mathrm{THz}$ emission experiments but becomes higher at lower ambient temperature if the pump fluence is increased. The time evolution of $\Delta R / R$ is mainly determined by the thermalization of the electrons with the phonon bath within $\mathrm{Pt}$, and we extract the electron-phonon thermalization time $\tau_{\mathrm{e}-\mathrm{ph}}=260 \pm 10 \mathrm{fs}$ from the fitting with $\exp \left(-\frac{t}{\tau_{\mathrm{e}-\mathrm{ph}}}\right)$. Although no quantitative conclusions can be drawn on the exact magnitude of temperature change, we can use the transient reflectivity measurements to conclude that $\Delta \mathrm{T}_{\mathrm{em}}$ only marginally depends on ambient temperature and cannot account for the temperature dependence of $S_{-}$. For $\Delta \mathrm{T}_{\mathrm{em}}$, we use the value of $200 \mathrm{~K}$ calculated in Ref. 18 for a similar device and similar experimental conditions.
Apart from $g^{\uparrow \downarrow}$, which quantifies the quality of the interface in conducting spins, the other parameters $\left(\lambda_{\mathrm{s}}, \sigma_{\mathrm{Pt}}\right.$, and $\left.\Theta_{\mathrm{SH}}\right)$ are intrinsic to the Pt layer. To exclude the contribution of these transport parameters or any other contribution from the setup, we compare our LSSE results to a metallic $\mathrm{THz}$ spintronic emitter ${ }^{20,35} \mathrm{CoFeB}(3 \mathrm{~nm}) \mid \mathrm{Pt}(5 \mathrm{~nm})$. In this case, the pump beam hits the sample from the $\mathrm{CoFeB}$ side and is largely absorbed by the ferromagnet, inducing a strong superdiffusive spin current. ${ }^{36,37}$ Therefore, far from $T_{\mathrm{C}}=1100 \mathrm{~K},{ }^{38} \mathrm{CoFeB}$ behaves as a temperature-independent spin current source, transported to the Pt layer by high mobility majority spin carriers. Equation (1) also applies to this metallic bilayer as it relies on the spin-to-charge conversion in Pt to generate $\mathrm{THz}$ emission. In agreement with a previous report, ${ }^{39}$ the amplitude of the $\mathrm{THz}$ pulse decreases with decreasing temperature and reaches a plateau at $50 \mathrm{~K}$ [Fig. 3(d)]. This behavior, which is associated with the intrinsic components of the spin Hall effect in $\mathrm{Pt}^{39}$ significantly differs to what is observed in our YIG|Pt sample, allowing us to exclude the influence of the Pt layer in our measured temperature dependence of the LSSE. We conclude, therefore, that our measurement probes the temperature dependence of the spin mixing conductance. 
The laser-excited free electrons in Pt are not spin polarized initially. The stochastic local exchange field fluctuations induced by single electron scattering events off the interface with the MI are therefore averaged to zero at timescales longer than the interaction time ( $\sim 4 \mathrm{fs}$ for $\mathrm{YIG} \mid \mathrm{Pt}^{18}$ ). Higher order interactions between the scattering electrons and the MI can lead to a net magnetic torque on the MI and therefore to spin accumulation, as described in Ref. 18. An additional contribution associated with the real part of the spin-mixing conductance $g^{r}$ is given by inelastic spin-flip scattering processes that result in the excitation of a magnon on the MI side. This contribution depends on the density of states of magnons as well as the electronic density of states at $T_{e}$. Using Eqs. (1) and (2), we estimate the range of the spin mixing conductance at $10 \mathrm{~K}$ as $g^{\uparrow \downarrow}=(1.8-8.4) \times 10^{18} \mathrm{~m}^{-2}$, in agreement with that found in Ref. 14. Our parameters are $Z_{0}=377 \Omega$, $n_{\mathrm{YIG}}=5,{ }^{18} n_{\mathrm{o}}=1, \sigma_{\mathrm{Pt}}(10 \mathrm{~K})=0.03 \mu \Omega^{-1} \mathrm{~cm}^{-1,37} \lambda_{\mathrm{s}}(10 \mathrm{~K})$ $=2-4 \mathrm{~nm},{ }^{40,41} \Theta_{\mathrm{SH}}=0.01-0.0223,{ }^{40} \quad M_{\mathrm{s}} \quad(10 \mathrm{~K})=172 \mathrm{kA} / \mathrm{m}$, $V=a^{3}, a=1.24 \mathrm{~nm}^{42}$ and $T_{\mathrm{e}}-T_{\mathrm{m}} \approx 200 \mathrm{~K}^{18}$ Note that $n_{\mathrm{YIG}}$ and $\sigma_{\mathrm{Pt}}$ can be considered frequency-independent within our detection bandwidth. ${ }^{27,43}$ We associate the kink in the temperature dependence of the LSSE signal around $80 \mathrm{~K}$ [Fig. 3(b)] with the population of the higher energy magnon bands in YIG by electron-magnon scattering with the highly energetic electrons in Pt. At an ambient temperature of $100 \mathrm{~K}$, the first high-frequency bands appear at $\sim 25 \mathrm{meV},{ }^{44-46}$ which coincides with the average energy of the optically heated electrons in Pt. The progressive filling of these bands at higher ambient temperature affects the spin pumped across the interface and determines the temperature dependence of the LSSE.

In conclusion, we characterize the low temperature behavior of the picosecond spin Seebeck effect in YIG|Pt by optical pump$\mathrm{THz}$ emission measurents and show that it is substantially different from that reported in low-frequency electrical measurements. We observe a sustained increase in the signal with decreasing temperature, which is a continuation of the previous femtosecond spin Seebeck effect experiment measured from room temperature to above $T_{\mathrm{c}}=550 \mathrm{~K}$. This behavior cannot be attributed to a variation of the temperature gradient at the interface or of the spin and charge transport characteristics in Pt and is instead to be associated with the spin-mixing conductance, providing direct access to its temperature dependence.

See the supplementary material for a discussion on the linear proportionality of $\Delta R / R$ on $\Delta \mathrm{T}_{\mathrm{e}}$ and the weak temperature dependence of transient reflectance in $\mathrm{Pt}$.

F.N.K. acknowledges support from the Cambridge Trust and the Jardine Foundation. C.C. and D.H. acknowledge support from the Winton Programme for the Physics of Sustainability. J.P. acknowledges support from the EPSRC. M.C., D.B., F.M., and M.T. acknowledge support from the Deutsche Forschungsgemeinschaft through the International Collaborative Research Center TRR160 (Project Nos. B8 and B9). C.C. and J.B. acknowledge support from the Royal Society. This work was supported by the COST Action MAGNETOFON (Grant No. CA17123).

\section{DATA AVAILABILITY}

The data that support the findings of this study are available from the corresponding author upon reasonable request.

\section{REFERENCES}

${ }^{1}$ K. Uchida, H. Adachi, T. Ota, H. Nakayama, S. Maekawa, and E. Saitoh, Appl. Phys. Lett. 97, 172505 (2010).

${ }^{2}$ K. Ando, S. Takahashi, J. Ieda, Y. Kajiwara, H. Nakayama, T. Yoshino, K. Harii, Y. Fujikawa, M. Matsuo, S. Maekawa, and E. Saitoh, J. Appl. Phys. 109, 103913 (2011).

${ }^{3}$ K. Uchida, J. Xiao, H. Adachi, J. Ohe, S. Takahashi, J. Ieda, T. Ota, Y. Kajiwara, H. Umezawa, H. Kawai, G. E. W. Bauer, S. Maekawa, and E. Saitoh, Nat. Mater. 9, 894-897 (2010).

${ }^{4}$ H. Adachi, K. Uchida, E. Saitoh, and S. Maekawa, Rep. Prog. Phys. 76, 036501 (2013).

${ }^{5}$ K. Uchida, T. Ota, H. Adachi, J. Xiao, T. Nonaka, Y. Kajiwara, G. E. W. Bauer, S. Maekawa, and E. Saitoh, J. Appl. Phys. 111, 103903 (2012).

${ }^{6}$ S. M. Wu, W. Zhang, A. KC, P. Borisov, J. E. Pearson, J. S. Jiang, D. Lederman,

A. Hoffmann, and A. Bhattacharya, Phys. Rev. Lett. 116, 097204 (2016).

${ }^{7}$ S. Seki, T. Ideue, M. Kubota, Y. Kozuka, R. Takagi, M. Nakamura, Y. Kaneko, M. Kawasaki, and Y. Tokura, Phys. Rev. Lett. 115, 266601 (2015).

${ }^{8}$ S. M. Wu, J. E. Pearson, and A. Bhattacharya, Phys. Rev. Lett. 114, 186602 (2015).

${ }^{9}$ D. Qu, S. Y. Huang, J. Hu, R. Wu, and C. L. Chien, Phys. Rev. Lett. 110, 067206 (2013).

${ }^{10}$ T. Kikkawa, K. Uchida, Y. Shiomi, Z. Qiu, D. Hou, D. Tian, H. Nakayama, X.-F. Jin, and E. Saitoh, Phys. Rev. Lett. 110, 067207 (2013).

${ }^{11}$ K. Uchida, T. Kikkawa, A. Miura, J. Shiomi, and E. Saitoh, Phys. Rev. X 4, 041023 (2014).

${ }^{12}$ E.-J. Guo, J. Cramer, A. Kehlberger, C. A. Ferguson, D. A. MacLaren, G. Jakob, and M. Kläui, Phys. Rev. X 6, 31012 (2016).

${ }^{13}$ A. Kehlberger, U. Ritzmann, D. Hinzke, E.-J. Guo, J. Cramer, G. Jakob, M. C. Onbasli, D. H. Kim, C. A. Ross, M. B. Jungfleisch, B. Hillebrands, U. Nowak, and M. Kläui, Phys. Rev. Lett. 115, 096602 (2015).

${ }^{14}$ T. Kikkawa, K. Uchida, S. Daimon, Z. Qiu, Y. Shiomi, and E. Saitoh, Phys. Rev. B 92, 064413 (2015).

${ }^{15}$ H. Jin, S. R. Boona, Z. Yang, R. C. Myers, and J. P. Heremans, Phys. Rev. B 92, 054436 (2015).

${ }^{16}$ S. M. Rezende, R. L. Rodríguez-Suarez, R. O. Cunha, A. R. Rodrigues, F. L. A. Machado, G. A. Fonseca Guerra, J. C. Lopez Ortiz, and A. Azevedo, Phys. Rev. B 89, 014416 (2014).

${ }^{17}$ M. Schreier, A. Kamra, M. Weiler, J. Xiao, G. E. W. Bauer, R. Gross, and S. T. B. Goennenwein, Phys. Rev. B 88, 094410 (2013).

${ }^{18}$ T. S. Seifert, S. Jaiswal, J. Barker, S. T. Weber, I. Razdolski, J. Cramer, O. Gueckstock, S. F. Maehrlein, L. Nadvornik, S. Watanabe, C. Ciccarelli, A. Melnikov, G. Jakob, M. Münzenberg, S. T. B. Goennenwein, G. Woltersdorf, B. Rethfeld, P. W. Brouwer, M. Wolf, M. Kläui, and T. Kampfrath, Nat. Commun. 9, 2899 (2018).

${ }^{19}$ J. Kimling, G.-M. Choi, J. T. Brangham, T. Matalla-Wagner, T. Huebner, T. Kuschel, F. Yang, and D. G. Cahill, Phys. Rev. Lett. 118, 057201 (2017).

${ }^{20}$ T. Seifert, S. Jaiswal, U. Martens, J. Hannegan, L. Braun, P. Maldonado, F. Freimuth, A. Kronenberg, J. Henrizi, I. Radu, E. Beaurepaire, Y. Mokrousov, P. M. Oppeneer, M. Jourdan, G. Jakob, D. Turchinovich, L. M. Hayden, M. Wolf, M. Münzenberg, M. Kläui, and T. Kampfrath, Nat. Photonics 10, 483-488 (2016).

${ }^{21}$ M. A. Ellabban, M. Fally, R. A. Rupp, and L. Kovács, Opt. Express 14, 593-602 (2006).

${ }^{22}$ S. Geller and M. A. Gilleo, J. Phys. Chem. Solids 3, 30-36 (1957).

${ }^{23}$ R. V. Pisarev, B. B. Krichevtsov, V. N. Gridnev, V. P. Klin, D. Frohlich, and C. Pahlke-Lerch, J. Condens. Matter Phys. 5, 8621-8628 (1993).

${ }^{24}$ M. Agrawal, V. I. Vasyuchka, A. A. Serga, A. Kirihara, P. Pirro, T. Langner, M. B. Jungfleisch, A. V. Chumak, E. T. Papaioannou, and B. Hillebrands, Phys. Rev. B 89, 224414 (2014).

${ }^{25}$ M. Schreier, F. Kramer, H. Huebl, S. Geprägs, R. Gross, S. T. B. Goennenwein, T. Noack, T. Langner, A. A. Serga, B. Hillebrands, and V. I. Vasyuchka, Phys. Rev. B 93, 224430 (2016).

${ }^{26}$ J. Xiao, G. E. W. Bauer, K. Uchida, E. Saitoh, and S. Maekawa, Phys. Rev. B 81, 214418 (2010).

${ }^{27}$ T. S. Seifert, N. M. Tran, O. Gueckstock, S. M. Rouzegar, L. Nadvornik, S. Jaiswal, G. Jakob, V. V. Temnov, M. Münzenberg, M. Wolf, M. Kläui, and T. Kampfrath, J. Phys. D 51, 364003 (2018).

${ }^{28}$ L. J. Krayer, J. Kim, and J. N. Munday, Opt. Mater. Express 9, 330 (2019). 
${ }^{29}$ D. L. Wood and K. Nassau, Appl. Opt. 29, 3704 (1990).

${ }^{30} \mathrm{~K}$. Lal and H. K. Jhans, J. Phys. C 10, 1315 (1977).

${ }^{31}$ S. A. Bender and Y. Tserkovnyak, Phys. Rev. B 91, 140402(R) (2015).

${ }^{32}$ A. N. Smith and P. M. Norris, Appl. Phys. Lett. 78, 1240 (2001).

${ }^{33}$ P. M. Norris, A. P. Caffrey, and R. J. Stevens, Rev. Sci. Instrum. 74, 400 (2003).

${ }^{34} \mathrm{M}$. Hase, K. Ishioka, J. Demsar, K. Ushida, and M. Kitajima, Phys. Rev. B 71, 184301 (2005).

${ }^{35}$ T. J. Huisman, R. V. Mikhaylovskiy, J. D. Costa, F. Freimuth, E. Paz, J. Ventura, P. P. Freitas, S. Blügel, Y. Mokrousov, T. Rasing, and A. V. Kimel, Nat. Nanotechnol. 11, 455-458 (2016).

${ }^{36} \mathrm{M}$. Battiato, K. Carva, and P. M. Oppeneer, Phys. Rev. Lett. 105, 027203 (2010).

${ }^{37}$ T. Kampfrath, M. Battiato, P. Maldonado, G. Eilers, J. Nötzold, S. Mährlein, V. Zbarsky, F. Freimuth, Y. Mokrousov, S. Blügel, M. Wolf, I. Radu, P. M. Oppeneer, and M. Münzenberg, Nat. Nanotechnol. 8, 256-260 (2013).
${ }^{38}$ H. Sato, P. Chureemart, F. Matsukura, R. W. Chantrell, H. Ohno, and R. F. L. Evans, Phys. Rev. B 98, 214428 (2018).

${ }^{39}$ M. Matthiesen, D. Afanasiev, J. R. Hortensius, T. C. van Thiel, R. Medapalli, E. E. Fullerton, and A. D. Caviglia, Appl. Phys. Lett. 116, 212405 (2020).

${ }^{40}$ M. Isasa, E. Villamor, L. E. Hueso, M. Gradhand, and F. Casanova, Phys. Rev. B 91, 024402 (2015).

${ }^{41}$ E. Sagasta, Y. Omori, M. Isasa, M. Gradhand, L. E. Hueso, Y. Niimi, Y. Otani, and F. Casanova, Phys. Rev. B 94, 060412(R) (2016).

${ }^{42}$ R. L. Douglass, Phys. Rev. 120, 1612-1614 (1960).

${ }^{43}$ A. M. Hofmeister and K. R. Campbell, J. Appl. Phys. 72, 638 (1992).

${ }^{44}$ J. Barker and G. E. W. Bauer, Phys. Rev. Lett. 117, 217201 (2016).

${ }^{45}$ A. J. Princep, R. A. Ewings, S. Ward, S. Tóth, C. Dubs, D. Prabhakaran, and A. T. Boothroyd, npj Quantum Mater. 2, 63 (2017).

${ }^{46}$ J. Barker, D. Pashov, and J. Jackson, Electron. Struct. 2, 044002 (2020). 